\title{
SIDEWALL CONTROL OF STATIC AZIMUTHAL BISTABLE NEMATIC ALIGNMENT STATES
}

\author{
S. Ladak ${ }^{\mathrm{a}}$, A. Davidson ${ }^{\mathrm{b}}$, C.V. Brown ${ }^{* a}$ and N.J. Mottram ${ }^{\mathrm{b}}$ \\ a School of Science and Technology, Nottingham Trent University, Erasmus Darwin \\ Building, Clifton Lane, Clifton, Nottingham, NG11 8NS United Kingdom. \\ ${ }^{\mathrm{b}}$ Department of Mathematics, University of Strathclyde, Livingstone Tower, \\ 26 Richmond Street, Glasgow G1 1XH, United Kingdom.
}

\begin{abstract}
Stable azimuthal alignment states have been created in the plane of a homogeneous layer of nematic liquid crystal by the action of one or more sawtooth sidewalls. The alignment states in devices with two sawtooth sidewall structures, either in-phase or in anti-phase, and with one sawtooth wall opposite a flat wall have been investigated as a function of the sawtooth pitch. The optical textures of the observed states are in excellent agreement with the predictions of nematic Q-tensor theory. The frequencies of occurrence of the different states are broadly consistent with the expected inverse correlation with the Q-tensor predictions for their energy.
\end{abstract}

\section{Keywords}

Nematic liquid crystal, surface effects, bistable states

*Author for correspondence:

Email carl.brown@ntu.ac.uk, Tel +44 (0)115 8483184, Fax +44 (0)115 8486636

This is the accepted version of paper which was subsequently published as follows:

"Sidewall Control of Static Azimuthal Bistable Nematic alignment states"

S. Ladak, A. Davidson, C.V. Brown and N.J. Mottram

J. Phys. D: Appl. Phys. Vol 42, pp85114-85121 (2009) 


\section{Introduction}

The creation of bistable and multistable orientations by use of a structured surface morphology on one or more of the bounding plates that confines a nematic liquid crystal layer has been the subject of increasing recent interest. Some of the morphologies that have been fabricated include microscale periodic arrays of posts or wells [1-5], surface steps [6], periodic gratings [1,7-9] and bigratings [10], and surface gratings created by nanoscale rubbing $[11,12]$. The different energetically stable states that are produced by the interaction between the nematic liquid crystal and the surface structures can occur at distinct zenithal out-of-plane tilt angles [1,6-8], at distinct azimuthal in-plane twist angles $[1,3,9,11,12]$, or at some combination of the two [2].

Multistable alignment has been demonstrated by the current authors in a simple square well geometry [4]. In that device, in-plane alignment, in which the nematic n-director is confined to the plane parallel to the bounding plates, has been created using substrates that were coated with photoresist layers that imparted degenerate planar anchoring. The nematic liquid crystal was laterally confined within square wells with sides of length between 4 and 6 times the layer thickness. For this range of aspect ratio the bounding plates maintain the in-plane alignment while the alignment at the edges of the square well influenced the whole of the well and reduces the tendency of the nematic to form a Schlieren texture [13]. These substrate-induced restrictions resulted in the formation of a small number of different azimuthal alignments of the 2-dimensional nematic n-director, that were consistent and controllable $[1,3,4]$. The nature of these azimuthal alignment states is determined almost entirely by the morphology of sidewalls of the wells which contain the liquid crystal, and not by the alignment properties of the upper and lower confining plates. These azimuthal alignment states can then be categorised by the position of defects, which tend to lie close to the corners of the square wells.

In the current work static nematic liquid crystal alignment configurations have been investigated both experimentally and theoretically in channels that are bounded by one or more in-plane sawtooth grating structures. This work follows from the realisation that any arbitrary topology of sidewall structure that can be fabricated using standard photolithography on the micrometer scale can be used to create lateral confinement. Since this geometry also allows the stable states and the underlying 2-dimensional director configurations to be simply and unambiguously visualised using polarised microscopy, it provides an excellent model system for studying the stabilising influence of nematic defects on the occurrence of bistable and multistable alignment configurations.

\section{Device construction}

The device structures that were used in this investigation are shown in figure 1, with the standard representation of a nematic liquid crystal layer, a cross section through the device in the yz-plane, shown in figure 1(a). A layer of nematic liquid crystal (shown by the ellipses representing the liquid crystal director) having thickness $w=15 \mu \mathrm{m}$ was confined in the z-direction between two glass substrates that had been coated with a layer of SU8 photoresist (MicroChem Corp., MA, USA), shown in figure 1(a) by the stripe pattern. Three different sidewall morphologies were used to laterally confine the nematic liquid crystal in the $x y$-plane as shown in black in figures 1(b), 1(c) and 1(d). The mean distance between the sidewalls was fixed at $d=80 \mu \mathrm{m}$. The sidewalls were also fabricated from SU8 photoresist and they formed open channels in the device along the $x$-direction. The devices contained an array of many adjacent channels (not shown in figure 1) along the $y$ - 
direction so that statistical analysis of the frequency of occurrence of optical textures in an area of the device with the same value of the period $p$ was possible.

Three different morphologies were investigated: sawtooth gratings on both sides of the well that were $180^{\circ}$ out of phase, as shown in figure 1(b); sawtooth gratings on both sides of the well in-phase with each other, as shown in figure 1(c); and a sawtooth grating opposite to a flat surface on the other side of the well, as shown in figure 1(d). The peak to trough amplitude of the sawtooth shaped sidewalls was fixed at $a=20 \mu \mathrm{m}$. For the remainder of this paper the geometries in figure 1(b), 1(c) and 1(d) will be referred to as sawtooth/sawtooth (antiphase), sawtooth/sawtooth (in-phase), and sawtooth/flat respectively.

The devices were fabricated using standard photolithography techniques and filled with the commercial nematic material E7 (Merck KGaA, Darmstadt, Germany). The process conditions and the filling technique are the same as those used for closed well structures that were described in [14]. After filling, the devices were put through a heating-cooling cycle to ensure that the positions of the different alignment states were changed by thermal annealing. This demonstrated that the regions that were affected by pinning of nematic defects near to unwanted inhomogeneities in the SU8 photoresist layer were small relative to the area of the devices that were studied.

For the range of pitch values that were used in the experimental devices it is not challenging to create sharp features in the sidewalls. Before filling, these features were observed at a magnification of $500 \times$ and the sawtooth structures were shown to be reproduced accurately by the photolithography process. Devices that showed significant overspill, where the nematic liquid crystal leaked over the wall areas, were not used for the investigations. In those devices the overspill caused pinning and it was found that the boundary regions between different states and certain defects and disclinations could not be moved or removed by thermal annealing.

\section{Theoretical model and simulation results}

In order to model the director configuration within the device we have developed a numerical methodology in which the liquid crystal alignment is modelled by the Q-tensor description and the minimisation of the total free energy (including terms describing the elastic, surface and thermotropic energies). This approach has been described in [15] and is thought to accurately model not only the director orientation, but also the change in order close to defects. It is the presence of defects in the current device which therefore necessitates such an approach. However, since such Q-tensor models are usually extremely computationally intensive we have also developed a method for approximating the n-director configuration which we can then use as an initial guess for the more accurate Q-tensor solution.

The Q-tensor approach used in this paper uses numerical minimisation, a fixed mesh finite element based adaptive time iteration scheme which evolves to an energy minimum, of the free energy in equation 1. 


$$
\begin{aligned}
F & =\int_{V}\left[\left(\frac{L_{1}}{2}\left(\frac{\partial Q_{i j}}{\partial x_{k}}\right)^{2}+\frac{L_{2}}{2} \frac{\partial Q_{i j}}{\partial x_{j}} \frac{\partial Q_{i k}}{\partial x_{k}}\right)+a_{b} \operatorname{tr}\left(\boldsymbol{Q}^{2}\right)+\frac{2}{3} b_{b} \operatorname{tr}\left(\boldsymbol{Q}^{3}\right)+\frac{1}{2} c_{b} \operatorname{tr}\left(\boldsymbol{Q}^{2}\right)^{2}\right] \mathrm{d} v \\
& +\int_{S}\left[c_{3} \boldsymbol{v} \cdot \boldsymbol{Q}^{2} \cdot \boldsymbol{v}+a_{S} \operatorname{tr}\left(\boldsymbol{Q}^{2}\right)+\frac{2}{3} b_{S} \operatorname{tr}\left(\boldsymbol{Q}^{3}\right)+\frac{1}{2} c_{S} \operatorname{tr}\left(\boldsymbol{Q}^{2}\right)^{2}\right] d s
\end{aligned}
$$

where $L_{1}$ and $L_{2}$ are elastic constants, related to the Frank elastic constants by $L_{1}=\left(K_{3}-K_{1}+3 K_{2}\right) /\left(6 S_{e}^{2}\right)$ and $L_{1}=\left(K_{1}-K_{2}\right) / S_{e}^{2}$ and $S_{e}$ is the order parameter at which the Frank elastic constants were measured. The parameters $a_{b}, b_{b}$ and $c_{b}$ are the bulk thermotropic parameters which determine the minimum energy state of the liquid crystal and the order parameter in the bulk of the system (i.e. zero for the isotropic state and greater than zero for the nematic state). The n-director prefers to lie tangential to the sidewalls due to the planar degenerate anchoring on the SU8 photoresist from which they are fabricated and this type of surface anchoring condition was used when calculating the director configurations that are depicted in figure 2. The surface anchoring parameter $c_{3}$ is related to the standard anchoring strength by $W_{s}=2 S_{e}^{2} c_{3} / 3$ and the surface thermotropic parameters $a_{s}, b_{s}$ and $c_{S}$ allow the boundary order parameter to be influenced independent of the director at the boundary.

As stated above, direct minimisation of this energy, without a sufficiently accurate initial guess for the solution of $\boldsymbol{Q}$ is computationally intensive. However, under certain simplifying assumptions a very good initial guess can be found for the director profile. If we assume that the order within the liquid crystal is constant (so that the eigenvalues of $\boldsymbol{Q}$ are constant), that the nematic director can be written as $\mathbf{n}=(\cos (\theta), 0, \sin (\theta))$ so that $\theta=\theta(x, y)$ is the angle between the director and the $x$ axis. If the liquid crystal is elastically isotropic, so that the elastic constants are equal, then the governing equation for the director structure reduces to $\nabla^{2} \theta=0$, Laplace's equation. In a two dimensional polygonal region Laplace's equation can be solved using conformal mapping, in this case using a Schwarz-Christoffel transformation [15-17]. In this way a large number of potential director configuration were modelled by using differing values of $\theta$ at the boundary walls.

However, it should be remembered that this conformal mapping approach will only give an approximation to the true director profile in the real device because we have made assumptions about the boundary conditions, the elastic constants and the order. To produce accurate director profiles, as shown in figure 2, we used the Q-tensor method described above, with the approximate solution used to construct an initial guess, $\boldsymbol{Q}=S_{e}(\boldsymbol{n} \otimes \boldsymbol{n}-\boldsymbol{I} / 3)$. To minimize the free energy in equation 1 from this initial guess we use the software package COMSOL Multiphysics (COMSOL Ltd, Hatfield, U.K.). For this numerical method we have used the elastic constants $K_{1}=11.1 \mathrm{pN}, K_{2}=6.5 \mathrm{pN}$, $K_{3}=17.1 \mathrm{pN}$, the anchoring strength $W_{S}=2 \times 10^{3} \mathrm{Nm}^{-1}, S_{e}=0.6$, and the thermotropic coefficients $\quad a_{b}=-0.112 \times 10^{6} \mathrm{Nm}^{-2}, \quad b_{b}=-0.64 \times 10^{6} \mathrm{Nm}^{-2}, \quad c_{b}=1.0 \times 10^{6} \mathrm{Nm}^{-2}$, $a_{s}=-8.524 \times 10^{3} \mathrm{Nm}^{-1}, b_{s}=-48.7 \times 10^{3} \mathrm{Nm}^{-1}$ and $c_{s}=76.1 \times 10^{3} \mathrm{Nm}^{-1}$. The director configuration may then be recovered from the Q-tensor solution by associating $\boldsymbol{n}$ with the eigenvector of $\boldsymbol{Q}$ corresponding to the largest eigenvalue of $\boldsymbol{Q}$ [18-20].

Figure 2 shows theoretical 2 dimensional director configurations for the 3 geometries: the alignment states A1, A2, A3 and A4 were obtained for the sawtooth/sawtooth (antiphase) geometry, the alignment states B1, B2, B3 and B4 for the sawtooth/sawtooth (in phase) 
geometry, and the alignment states C1, C2 and C3 for the sawtooth/flat geometry. Within an individual $\mathrm{V}$-shaped groove of the sawtooth sidewall two distinct configurations can be identified which will be referred to "vertical" or "horizontal defect-free" near-sidewall alignment patterns, following the nomenclature of reference [1].

The horizontal defect-free alignment pattern is exhibited at all of the sawtooth sidewalls in the states labelled A1, B1, C1, A2, B2, and C2 in figure 2. For all of these states, close to the sidewalls, the nematic n-director follows the contours of the sawtooth and there are no nematic defects. In states A1, B1 and C1 the director distortion decays smoothly into the bulk away from the sidewall and in the central region of the channel the director is substantially horizontally aligned. In states A2, B2 and C2 the director in the central region of the channel is vertically aligned and so the director rotates by $\pi$ radians on moving across the channel from the upper wall to the lower sidewall. In the y-direction this is reminiscent of a bend state, as observed in a pi-cell geometry.

The vertical alignment pattern is exhibited at all the sawtooth sidewalls in the states labelled A3, B3, C3, A4 and B4 in figure 2. For all of these states there are nematic defects in the vicinity of the peaks and troughs of the sawtooth sidewall structures. In states A3 and B3 the director in the central region of the channel is horizontally aligned and so the director rotates by $\pi$ radians on moving across the channel from the upper wall to the lower sidewall. In the y-direction this is reminiscent of a splay alignment state. In the state labelled C3 there is horizontal alignment at the flat upper sidewall and vertical alignment at the lower sawtooth giving a rotation of the director by $\pi / 2$ radians on moving from the upper to the lower sidewall, reminiscent of a HAN (Hybrid Aligned Nematic) state in the y-direction. In states A4 and B4 the director distortion decays smoothly into the bulk away from the sidewall and the central region of the channel is substantially vertically aligned.

There are other possible alignment configurations, which will not be further considered, in which the horizontal defect-free alignment pattern is exhibited one of the sawtooth sidewalls and the vertical alignment pattern is exhibited at the other sidewall.

In order to compare the theoretical director configurations shown in figure 2 to experimental optical textures it was necessary to calculate the optical transmission of the liquid crystal orientation when placed between crossed polarisers. For this calculation, since we have assumed that the director remains in the $x y$-plane, we have also assumed that light incident perpendicular to the bounding plates (i.e. in the $z$-direction), remains perpendicular to the bounding plates throughout the device and, therefore, that the transmission at the point $(x, y)$ is only a function of the director angle at that point. The optical transmission is then $T=\sin ^{2}(2 \chi) \sin ^{2}(\delta / 2)$ [21], where $\delta=\left(2 \pi n_{O} n_{e} w / \lambda\right)\left(\left[n_{e}^{2}+\left(n_{O}^{2}-n_{e}^{2}\right) \cos ^{2} \theta\right]^{-1 / 2}-1\right)$ is the retardation of the liquid crystal and $\chi$ is the angle the director makes with either polariser. In this expression $w$ is the cell thickness, see figure 1(a), the wavelength of light was taken to be $\lambda=600 \mathrm{~nm}$ and the optical parameters for the liquid crystal used in the experiment were: ordinary refractive index $n_{O}=1.5$; extraordinary refractive index $n_{e}=1.721$.

\section{Experimental Results}

Figures 3(a) and 3(b) show two distinct nematic alignment textures that were observed and modelled in the sawtooth/sawtooth (antiphase) geometry, figures 4(a) and 4(b) show two textures in the sawtooth/sawtooth (in phase) geometry, and figures 5(a), 5(b) and 5(c) 
show three textures in the sawtooth/flat geometry. In figures 3, 4 and 5 the sawtooth period is $p=60 \mu \mathrm{m}$. The images in each of the columns labelled "experiment" show optical textures that were observed in transmission with a polarising microscope at a magnification of $200 \times$. The polariser and the analyser were crossed at $90^{\circ}$ and the angles given in the figures refer to the angle between the polariser and the horizontal. In figures 3(a) and 3(b) the two optical textures shown were observed in distinct regions of the same device having the same sawtooth period. The two textures co-existed either in separate open channels in the array of parallel adjacent channels in the $y$-direction, or along the same channel but separated by a distorted region spanning the width of one or two periods of the sawtooth structure. This was also the case for the two textures observed in the other geometries, shown in figure 4 and shown in figure 5.

The images in the column labelled "theory" in figures 3, 4 and 5 show the theoretical optical textures for the same polariser orientations that were used when observing the experimental optical textures. These images were generated from the 2-dimensional nematic n-director alignment profiles depicted in figure 2 using the method described in section 3 above. The theoretical optical textures were generated using the following director configurations : A1 for 3(a), A2 for 3(b), B1 for 4(a), B2 for 4(b), C1 for 5(a), C2 for 5(b), and C3 for 5(c). There is excellent agreement between the experimental and theoretical optical textures. The appearance of the experimental optical textures between different orientations of the crossed polarisers allows unequivocal identification with particular theoretical 2 dimensional alignment configurations. For example the optical textures resulting from state A1 and A4 in figure 2 can be distinguished from each other because the dark brushes associated with the distorted regions near to the sawtooth sidewalls separate and rotate in opposite senses when the textures are rotated between crossed polarisers.

Figure 6(a) shows the experimental frequency of occurrence of the alignment states A1 and A2 in the sawtooth/sawtooth (antiphase) geometry as a function of the pitch $p$ of the sawtooth sidewall where the pitch was varied between $20 \mu \mathrm{m}$ and $100 \mu \mathrm{m}$ in steps of $10 \mu \mathrm{m}$. At each pitch value the number of full periods of the sawtooth was counted for which the optical texture of figure 3(a) was exhibited for A1, and for which the optical texture of figure 3(b) was exhibited for A2. Transition regions between the states and solitary defect textures were discounted from the total. The frequency of occurrence was calculated by dividing the total by the total number of periods at each pitch $p$. A similar process was used to obtain the experimental frequency of occurrence of the alignment states B1/4(a) and B2/4(b) for the sawtooth/sawtooth (in phase) geometry in figure 7(a) and C1/5(a), C2/5(b), and C3/5(c) for the sawtooth/flat sidewall geometry in figure 8(a). In each case the device underwent slow thermal annealing before the observations were made. This involved: heating at $1^{\circ} \mathrm{C}$ per minute to $70^{\circ} \mathrm{C}$ (which is well into the isotropic phase); leaving the sample for 15 minutes at $70^{\circ} \mathrm{C}$; cooling at less than $0.5^{\circ} \mathrm{C}$ per minute to room temperature, and then leaving the device for 12 hours to ensure no further defect reorientation was taking place. Thermal annealing was shown to randomise the occurrence of the different alignment states and to change the position of the boundary regions between different states, but statistics of the frequency of occurrence were not changed significantly by this process.

\section{Discussion}

Figure 6(b) shows the theoretical predictions for the energies of states A1, A2, A3 and A4 that were calculated at different values of the pitch p using Q-tensor theory with the method and the parameters described earlier in section 3. Figure 7(b) shows the results of 
similar calculations but for the alignment states B1, B2, B3 and B4 and figure 8(b) shows the theoretical energies for the alignment states C1, C2 and C3.

Consider the alignment states A1/3(a) and A2/3(b) from figures 2 and 3 for the sawtooth/sawtooth (antiphase) geometry. The theoretical energies in figure 6(b) show the following trends: at lower pitch values the energy of states A1 and A2 are comparable; as the pitch is increased the energy of both states decreases but the energy of state A1 as a fraction of the energy of state A2 decreases until it has less than $50 \%$ of the energy of state A2 when $p=100 \mu \mathrm{m}$. These observations are in broad agreement with the experimental results shown in figure 6(a). State A1 has a greater frequency of occurrence than state A2 for the whole range of pitch values investigated, which is consistent with state A1 having lower energy than state A2. At lower values of the pitch, where the energies of A1 and A2 are predicted to be comparable, the experimental frequencies of occurrence of states A1 and $\mathrm{A} 2$ are also more comparable.

Now consider the alignment states B1/4(a) and B2/4(b) from figures 2 and 4 for the sawtooth/sawtooth (in phase) geometry. The theoretical energies in figure 7(b) show the very similar trends for $\mathrm{B} 1$ and $\mathrm{B} 2$ as are shown by $\mathrm{A} 1$ and $\mathrm{A} 2$ in figure 6(b), i.e. the energy of states B1 and B2 are comparable at lower pitch values, but the energy of state B1 is proportionally much lower than for B2 at higher pitch values. In this geometry the experimental results are consistent with the predicted trend at high pitch values above $\sim 60 \mu \mathrm{m}$ : the lower energy B1 state becomes increasing more probable and the higher energy B2 state is observed less frequently as the pitch increases until at the highest pitch state B2 is no longer observed. At low pitches the experimental observations are not consistent with the energy predictions: state B2 shows a peak in probability at $60 \mu \mathrm{m}$ and for the lower pitches state B1 is observed over $90 \%$ of the area of the device.

In the sawtooth/flat geometry three states have been observed in an experimental device: C1/5(a), C2/5(b) and C3/5(c) from figures 2 and 5. From figure 8(a), state C1 is observed covering a large proportion of the area of the device for pitch values from $30 \mu \mathrm{m}$ up to the highest value investigated, $100 \mu \mathrm{m}$. The frequency of occurrence of state C1 only drops sharply at the lowest pitch value, $20 \mu \mathrm{m}$. The probability of occurrence of states C2 and C3 are very low: only occurring at intermediate pitch values with peaks at $60 \mu \mathrm{m}$ and $50 \mu \mathrm{m}$ respectively. The experimental observations for state $\mathrm{C} 1$ are broadly in agreement with the theoretical predictions of the energy in figure 8(c) where state C1 has the lowest energy for pitch values above $40 \mu \mathrm{m}$, but the energy of this state rises sharply at pitch $30 \mu \mathrm{m}$ and below this. State C3 has the lowest energy at pitch values below $40 \mu \mathrm{m}$, but this state shows very low area coverage of $1-2 \%$ at these pitch values.

For the states that are observed over more than $10 \%$ of the area of the experimental devices (A1, A2, B1, B2 and C1) the frequency of occurrence of these states are broadly consistent with the expected inverse correlation with the theoretical predictions of the energy of the states. For all of these states a horizontal defect-free alignment pattern is exhibited at sawtooth sidewalls, and this is combined either with horizontal alignment in the bulk of the channel for the most common states (A1, B1 and C1) or with vertical alignment in the bulk of the channel for the less common states (A2 and B2 "bend states"). The predicted crossover in energies at low pitches, where the states with the vertical alignment at the sawtooth sidewalls become more energetically favourable, is not observed in experimental devices. States A3, A4, B3, B4 were not observed, and state C3 was only observed in a narrow range of pitch values covering less than $5 \%$ of the area of the device. 
There are several reasons why the direct comparison between the experimental frequency of occurrence and the theoretical predictions for the energies of the states is not straightforward. The predicted energy of a given alignment state can be related inversely to the statistical probability that that state occurs in isolation. This does not give any information regarding the energy barriers between the different stable states. In an experimental device different states are often observed along the same channel separated by a transition region that contains elastic energy and, depending on whether the neighbouring states are topologically distinct, that may also contain a nematic defect. In addition, characteristic isolated defects that span the width of one period of the sawtooth sidewall structure have also been observed within regions that are otherwise in a single alignment state. These transition regions and defects can cover up to $20 \%$ of the area of the device. Regarding the geometry, the channels are open-ended and are connected to a reservoir of nematic liquid crystal material which is only confined in the z-direction and which therefore exhibits a Schlieren optical texture corresponding to degenerate planar anchoring on the upper and lower plates.

Studies of the dynamic switching between distinct stable states may elucidate the nature of the energy barrier between them and the results from these investigations will be addressed in a future publication.

\section{Acknowledgements}

The authors gratefully acknowledge the HEFCE for funding, through RAE funds allocated by Nottingham Trent University and the EPSRC for grants EP/F014988/1 and $\mathrm{EP} / \mathrm{F} 01502 \mathrm{X} / 1$. 


\section{References}

[1] R.N. Thurston, J. Cheng and G.D. Boyd, IEEE Trans. Elec. Dev. ED27(11), 2069 (1980)

[2] S. Kitson and A. Geisow, Appl. Phys. Lett. 80(19), 3635 (2002)

[3] N.J. Mottram, A. Ramage, G. Kelly and A.J. Davidson, UK patent GB20040026582, 8th June 2006

[4] C. Tsakonas, A. Davidson, C.V. Brown and N. J. Mottram, Appl. Phys. Lett. 90, 111913 (2007)

[5] A. Majumdar, C.J.P. Newton, J.M. Robbins and M. Zyskin, Phys. Rev. E. 75, 051703 (2007)

[6] C. Uche, S.J. Elston and L.A. Parry-Jones, J. Phys. D: Appl. Phys. 38, 2283 (2005)

[7] G.P. Bryan-Brown, C.V. Brown and J.C. Jones, UK Patent GB2318422, 16 Oct 1995.

[8] C.V. Brown, M.J. Towler, V.C. Hui and G.P. Bryan-Brown, Liq. Cryst. 27, 233 (2000).

[9] R. Barberi, J.J. Bovent, M. Giocondo, M. Iovane and A.L. Alexe-Ionescu, J. Appl. Phys. 84(3), 1321 (1998).

[10] G. P. Bryan-Brown, M.J. Towler, M.S. Bancroft, D.G. McDonnell, UK patent GB2286467, 8 Feb 1995

[11] J-H. Kim, M. Yoneya, J. Yamamoto and H. Yokoyama, Appl. Phys. Lett. 78(20), 3055, (2001)

[12] J-H. Kim, M. Yoneya, J. Yamamoto and H. Yokoyama, Nature 420, 159 (2002)

[13] S. Chandresekhar, "Liquid Crystals", p. 30, 2nd Ed. Cambridge University Press (1992)

[14] G.G. Wells and C.V. Brown, Appl. Phys. Lett. 91 p 223506 (2007)

[15] M.R. Spiegel, Complex Variables with an Introduction to Conformal Mapping and Its Applications, Schaum's Outlines (1999)

[16] T.A. Driscoll and L.N. Trefethen, Schwarz-Christoffel Mapping, Cambridge University Press (2002)

[17] V.I. Ivanov and M.K. Trubetskov, Handbook of Conformal Mapping with Computer-Aided Visualization, CRC Press (1994)

[18] P.G. DeGennes and J. Prost, The Physics of Liquid Crystals, Clarendon Press, Oxford, (1993)

[19] E.B. Priestly, P.J. Wojtowicz and P. Sheng, Introduction to liquid crystals, Plenum Press, New York and London (1976)

[20] E.K. Tidey, L.A. Parry-Jones and S.J. Elston, Liquid Crystals 34(2), 251-255 (2007)

[21] M. Bourne and H. Wolf, Principles of Optics - sixth edition, Pergamon Press (1980) 


\section{Figure captions}

Figure 1 The device used in the investigation: (a) shows a cross section through the device in the z-direction and (b), (c) and (d) show plan views of the different sidewall morphologies that were used to confine the nematic liquid crystal in the $x-y$ plane. The ellipses indicate the layer of nematic liquid crystal.

Figure 2 Theoretical 2-dimensional director profiles for the geometries shown in figures 1(b), 1(c) and 1(d) from Q-tensor theory. The alignment states A1, A2, A3 and A4 were obtained for the sawtooth/sawtooth (antiphase) geometry, alignment states B1, B2, B3 and B4 for the sawtooth/sawtooth (in phase) geometry, and alignment states C1, C2 and C3 for the sawtooth/flat geometry.

Figure 3 Experimental and theoretical optical textures for the device geometry in figure 1(b), the sawtooth/sawtooth (antiphase) geometry. 3(a) and 3(b) show two distinct experimental optical textures that were observed between crossed polarisers at several different orientations under a polarising microscope with a magnification of $200 \times$. The theoretical optical textures were generated using the following director configurations depicted in figure 2 : A1 for 3(a) and A2 for 3(b).

Figure 4 Experimental and theoretical optical textures for the device geometry in figure 1(c), the sawtooth/sawtooth (in phase) geometry. 4(a) and 4(b) show two distinct experimental optical textures that were observed between crossed polarisers at several different orientations under a polarising microscope with a magnification of $200 \times$. The theoretical optical textures were generated using the following director configurations depicted in figure 2 : B1 for 4(a) and B2 for 4(b).

Figure 5 Experimental and theoretical optical textures for the device geometry in figure 1(d), the sawtooth/flat geometry. 5(a), 5(b) and 5(c) show three distinct experimental optical textures that were observed between crossed polarisers at several different orientations under a polarising microscope with a magnification of $200 \times$. The theoretical optical textures were generated using the following director configurations depicted in figure 2 : C1 for 5(a), C2 for 5(b), and C3 for 5(c).

Figure $6 \quad$ (a) The experimental frequency of occurrence of the alignment states A1 and A2. (b) The theoretical predictions for the energies of states A1, A2, A3 and A4. In both plots the data are shown as a function of the pitch $p$ for the sawtooth/sawtooth (antiphase) geometry.

Figure $7 \quad$ (a) The experimental frequency of occurrence of the alignment states B1 and B2. (b) The theoretical predictions for the energies of states B1, B2, B3 and B4. In both plots the data are shown as a function of the pitch $p$ for the sawtooth/sawtooth (in phase) geometry.

Figure 8 (a) The experimental frequency of occurrence of the alignment states C1, C2 and C3. (b) The theoretical predictions for the energies of states C1, C2, 
C3 and C4. In both plots the data are shown as a function of the pitch $p$ for the sawtooth/flat geometry.

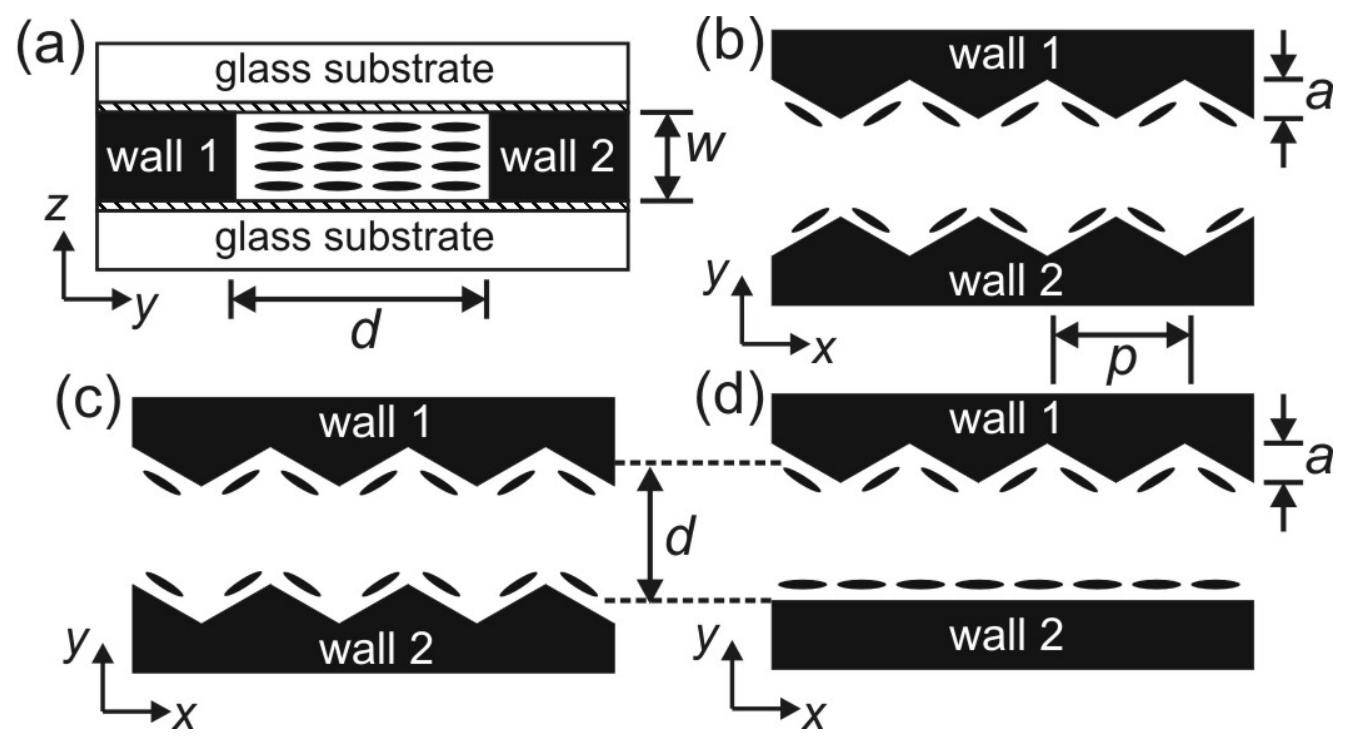

Figure 1. Ladak et. al. 

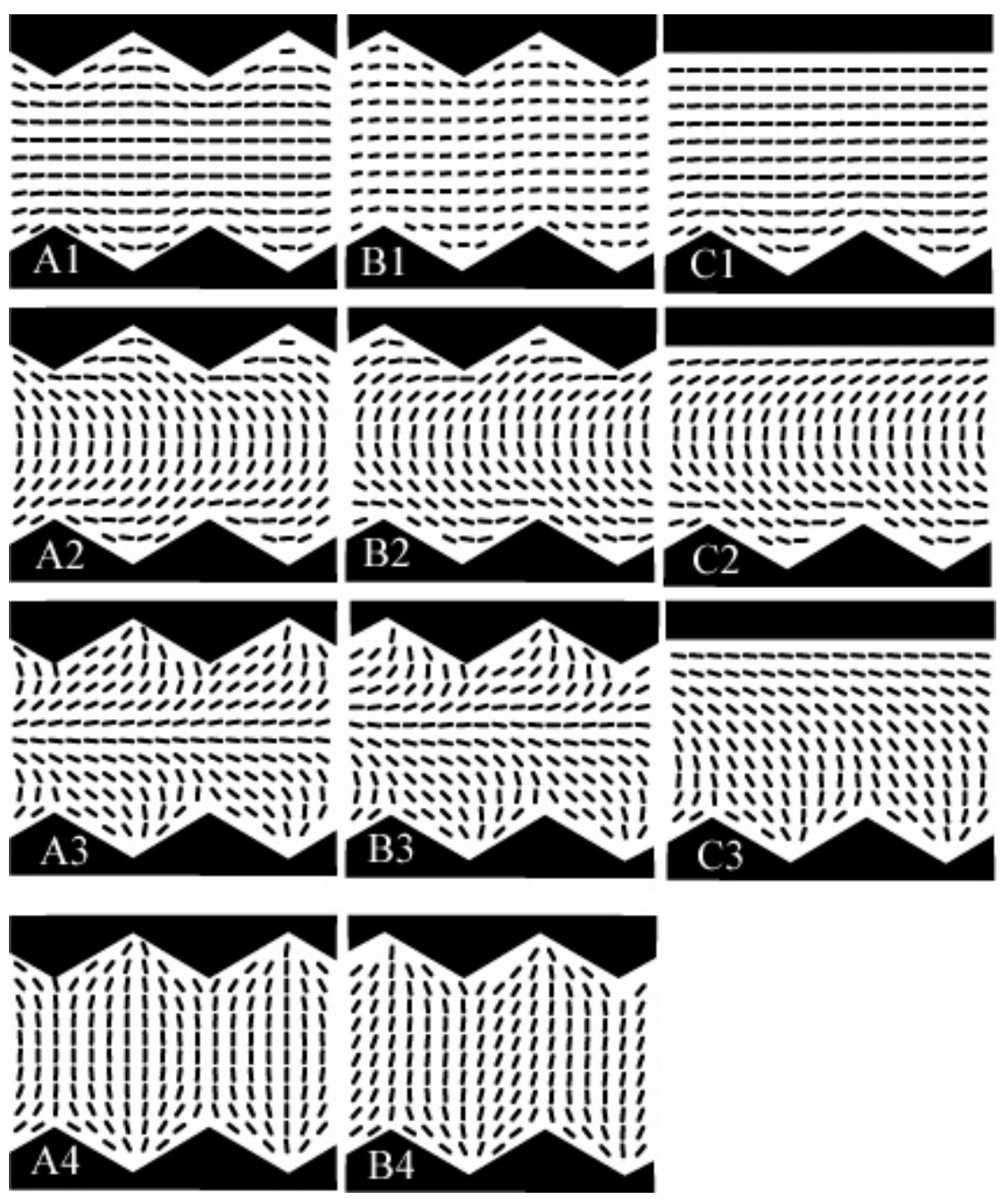

Figure 2. Ladak et. al. 
(a)

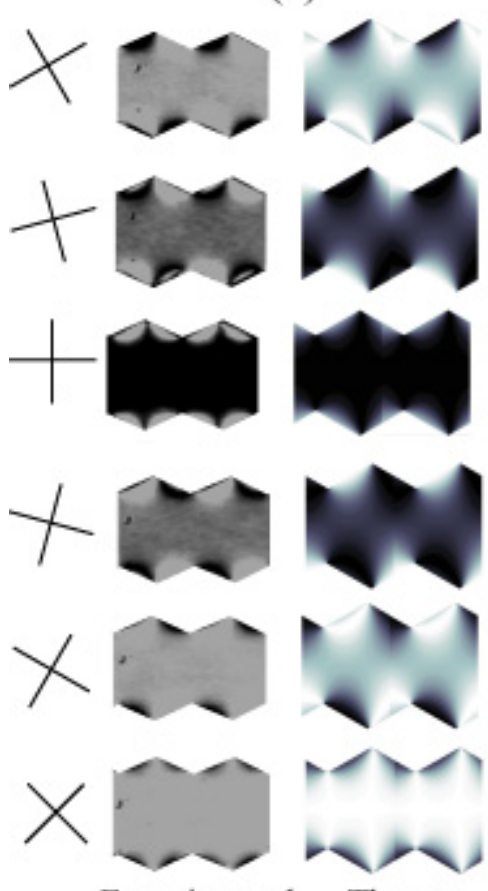

Experimental Theory (b)
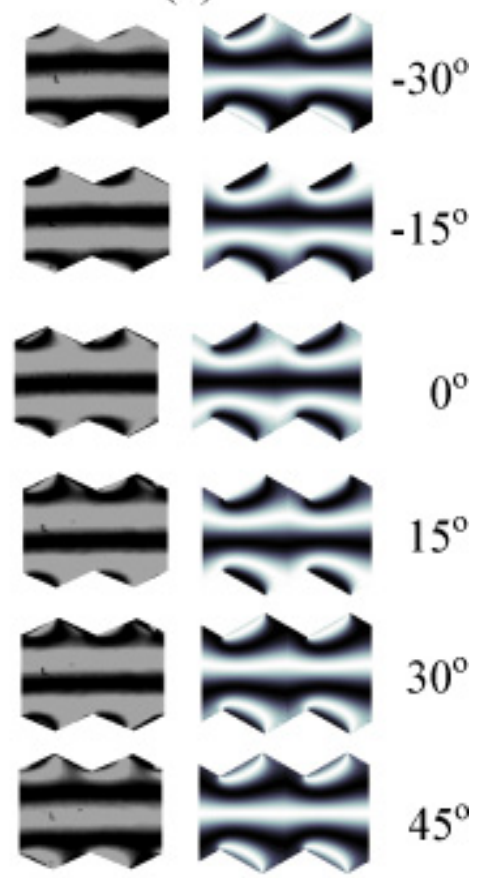

Experimental Theory

Figure 3. Ladak et. al. 
(a)

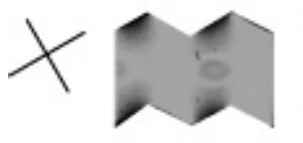

10

$1+$
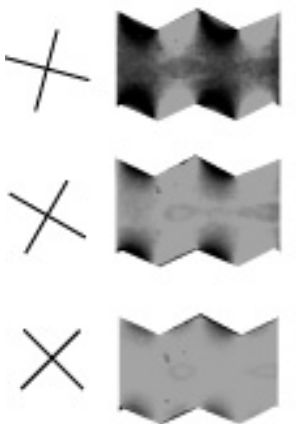

Experimental
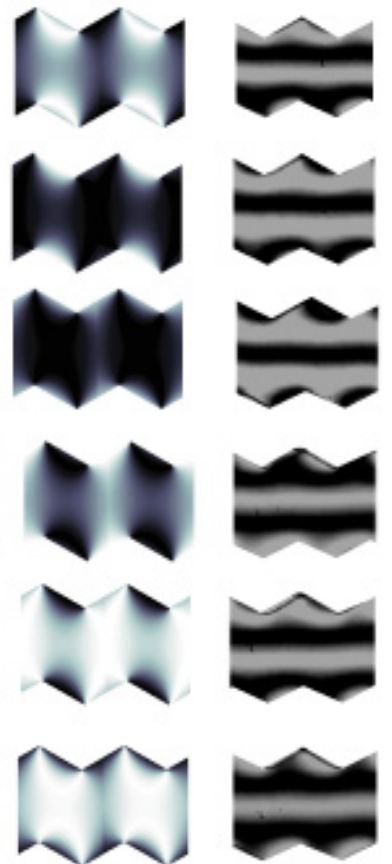

Theory (b)
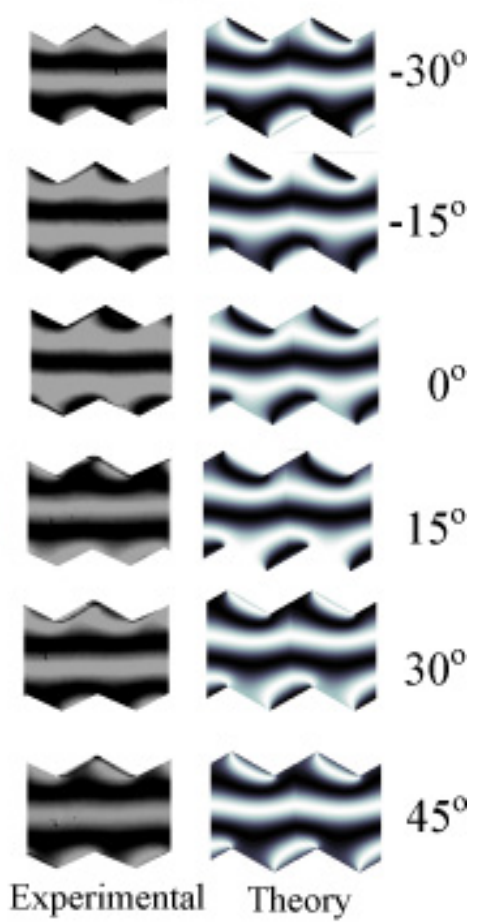

Figure 4. Ladak et. al. 
(a)

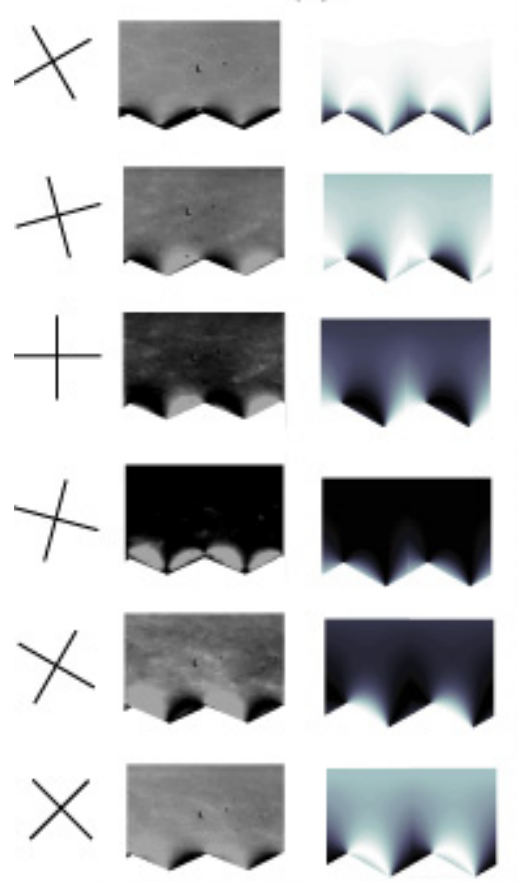

Experimental Theory (b)
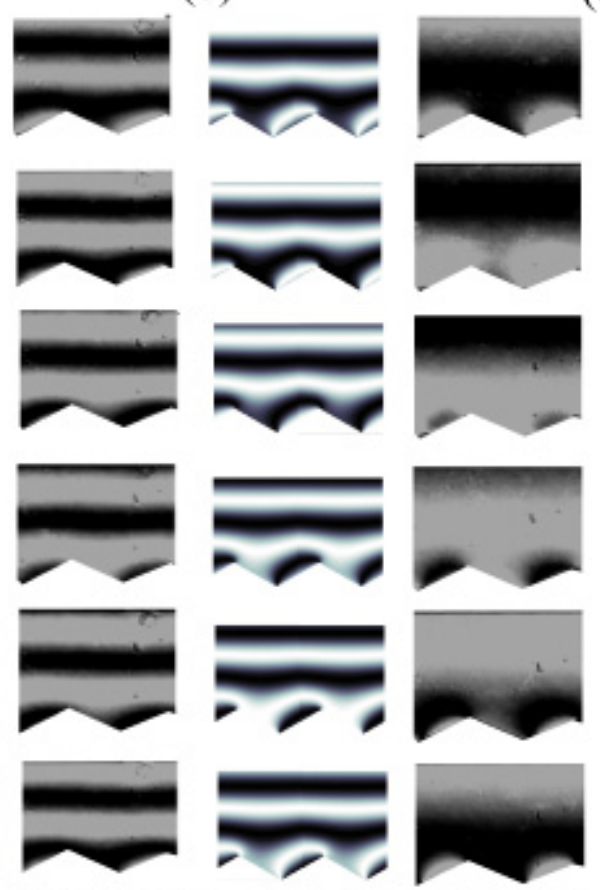

Experimental
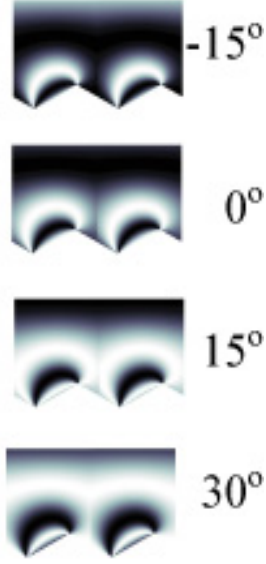

(c)
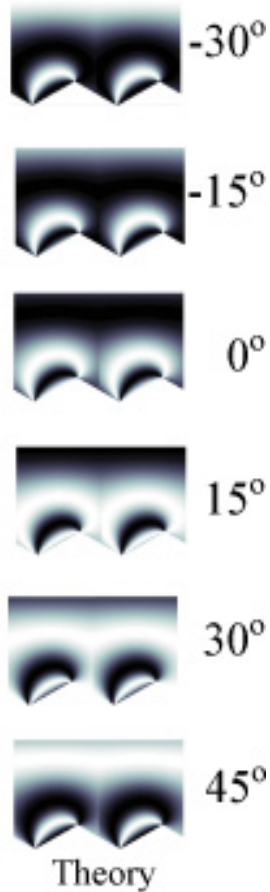

Figure 5. Ladak et. al. 

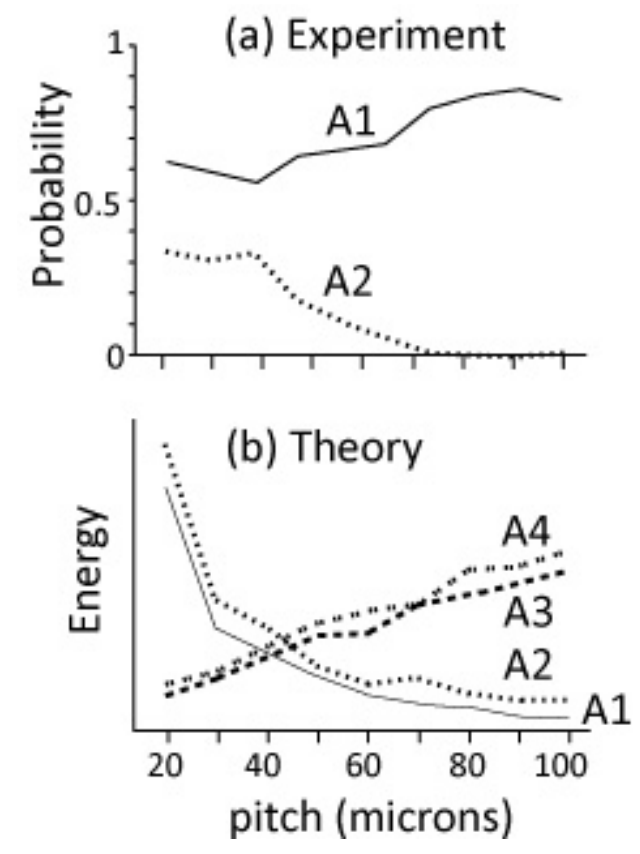

Figure 6. Ladak et. al. 

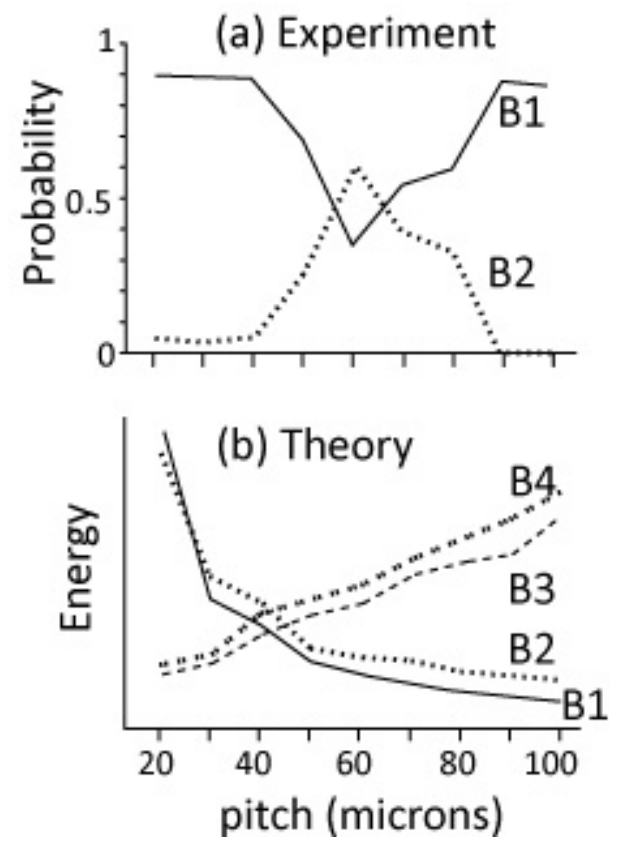

Figure 7. Ladak et. al. 

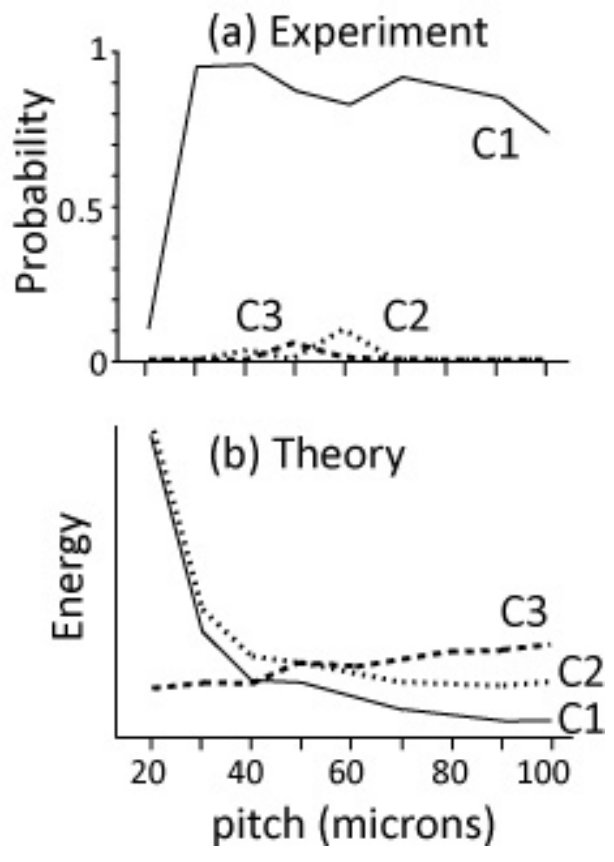

Figure 8. Ladak et. al. 